\title{
Klinikhygiene: Zielstandard noch nicht erreicht
}

— in Drittel der Krankenhäuser mit über - 400 Betten hat noch nicht den von der Kommission für Krankenhaushygi-

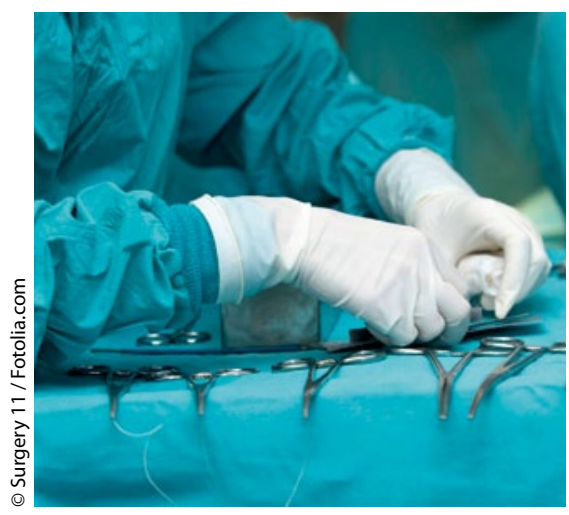

Im OP-Saal ist höchstmögliche Infektionspävention gefordert. ene und Infektionsprävention (KRINKO) empfohlenen Standard erreicht, wonach bei dieser Größenordnung ein hauptamtlicher Hygienefacharzt in der Klinik tätig sein soll. $\mathrm{N}$

Nach Angaben des Deutschen Krankenhausinstituts sind in großen Kliniken mit über 600 Betten im Schnitt 1,6 hauptamtliche Fachärzte für Hygiene tätig. In Krankenhäusern mit unter 600 Betten sind es lediglich 0,5 hauptamtliche Fachärzte. Ihre Arbeit wird zu mehr als der Hälfte durch organisatorische Tätigkeiten absorbiert.

$70 \%$ der Krankenhäuser haben inzwischen ein obligatorisches und standardisiertes MRSA-Aufnahmescreening installiert.

Helmut Laschet
Pflegeversicherung Demenz: Pflegebeirat neu konstituiert

Das Bundesgesundheitsministerium unternimmt einen neuen Anlauf, den Pflegebedürftigkeitsbegriff neu zu definieren. Dies war im vergangenen Jahr mangels präziser Vorgaben des Ministeriums gescheitert. Unter dem Vorsitz des Patientenbeauftragten der Bundesregierung, Wolfgang Zöller (CSU), und des ehemaligen Vorstandes des GKVSpitzenverbands, Klaus Dieter Voß, soll der Pflegebeirat nun die Details ausarbeiten, wie die Leistungen der Pflegeversicherung speziell für Demenzkranke ausgestaltet werden können. Dies ist im gegenwärtig existierenden Entwurf für die Reform der Pflegeversicherung noch ausgespart.

Helmut Laschet

\section{Schärfere Kontrollen für Medizinprodukte in der Diskussion}

\begin{abstract}
/ or dem Hintergrund der Gesundheitsrisiken ungeeigneter Brustimplantate der pleite gegangenen französischen Firma PIP wird nun auch in der Union über schärfere Kontrollen für Medizinprodukte der höchsten Risikostufe diskutiert. So schlägt CDU-Bundestagsabgeordneter Dietrich Monstadt vor, die Zulassung zu ändern: Benannte Stellen wie etwa der TÜV sollen sich nicht nur
\end{abstract}

auf Herstellerangaben verlassen, sondern müssten Implantate selbst auf deren Funktionalität und Sicherheit testen. Das müsse europaweit geregelt werden. Ferner sollen Ärzte - strafbewehrt - verpflichtet werden, sich an einem Medizinproduktebeobachtungs- und meldesystem zu beteiligen. Die deutsche Gesellschaft für plastische Chirurgie fordert gar ein Medizinprodukteregister.
Der GKV-Spitzenverband will, dass innovative Medizinprodukte zunächst nur in Innovationszentren unter systematischer Beobachtung eingesetzt werden. Das IQWiG plädiert für ein staatliches Zulassungsverfahren. Grünen-Gesundheitspolitikerin Birgitt Bender fordert eine frühe Nutzenbewertung für Medizinprodukte, die in den menschlichen Körper implantiert werden.

Helmut Laschet

\section{Achtung! Hier muss der Platzhalter durch eine Anzeige ersetzt werden!}

